\title{
A dual regimen of ritonavir/darunavir plus dolutegravir for rescue or simplification of rescue therapy: 48 weeks' observational data
}

\author{
Amedeo F. Capetti ${ }^{1 *}$, Maria Vittoria Cossu', Giancarlo Orofino², Gaetana Sterrantino ${ }^{3}$, Giovanni Cenderello 4 \\ Giuseppe V. De Socio ${ }^{5}$, Anna Maria Cattelann ${ }^{6}$, Alessandro Soria ${ }^{7}$, Stefano Rusconi ${ }^{8}$, Niccolò Riccardi ${ }^{9}$, \\ Gian Maria Baldin ${ }^{10}$, Fosca P. Niero ${ }^{1}$, Giorgio Barbarini ${ }^{11}$ and Giuliano Rizzardini ${ }^{1,12}$
}

\begin{abstract}
Background: Dolutegravir (DTG) plus darunavir/ritonavir (DRV/r) is a simple combination of drugs that has the best genetic barrier to HIV-1 resistance and may be fit for salvage therapy.

Methods: All HIV-1-infected subjects treated with DTG plus DRV/r between March 2014 and September 2015 in eight Italian centres were included in the analysis. The main metabolic data, efficacy parameters and safety data routinely collected were provided. This observational study is aimed to assess the efficacy of such approach. The primary endpoint was the proportion of subjects achieving or maintaining virologic suppression $<50$ copies $/ \mathrm{mL}$ at week 24 . Secondary end points were maintaining virologic suppression in the follow-up (weeks 48 and 96) and safety.

Results: One hundred and thirty subjects were followed for a median of 56 months. Reasons for switching were simplification (44.6\%), viral failure (30\%), toxicity (16.9\%), non-adherence (4.6\%), persistent low-level viremia (3.1\%), and drug-drug interaction (0.8\%). At baseline, 118 subjects had documented resistance to 1 to 5 antiretroviral classes while 12 had viral rebound at a time when genotypic tests were not yet available.

Seventeen and 14 subjects took DRV/r and DTG twice daily, respectively. One subject was lost to follow-up, one discontinued for liver enzymes' elevation, one died of illicit drug abuse and one of cancer-related complications. The proportion of subjects with ongoing HIV replication dropped from $40 \%$ to $6.1 \%$. Those with undetectable viral load increased from 38.5\% to 76.2\%. At week 48, 17.7\% had HIV RNA between 1 and 49 copies $/ \mathrm{mL}$.

The number of subjects with altered serum glucose, creatinine, ALT, AST, total-, HDL- and LDL-cholesterol, triglycerides and MDRD $<90 \mathrm{~mL} / \mathrm{min}$ decreased by week 48 , while those having MDRD $<60 \mathrm{~mL} /$ min remained 4.6\%. Overall 90/283 baseline laboratory alterations returned to normality.
\end{abstract}

Conclusions: Switching to DTG plus DRV/r proved to be safe, suppressing viral replication without metabolic impact.

Keywords: Dolutegravir, Darunavir, Ritonavir, Switch, Dual, Salvage, Simplification

\footnotetext{
* Correspondence: amedeo.capetti@unimi.it

Data from this work have been presented at the 14th European Meeting on HIV \& Hepatitis, 25-27 May 2016, Rome, Italy, at the HIV Drug Therapy Congress, 24-26 October 2016 Glasgow, UK and to the 9th Italian Conference on AIDS and Antiviral Research, 12-14 June 2017, Siena, Italy. ${ }^{1} 1$ st Division of Infectious Diseases, ASST Fatebenefratelli-Sacco, Via Giovanni Battista Grassi, 74, pavillion 56, Malattie Infettive, 2nd floor, 20157 Milan, Italy Full list of author information is available at the end of the article
} 


\section{Background}

The present work is the 48-week update of a previously reported study [1], concerning a retrospective-prospective follow-up of subjects who had been switched for any reason to a dual combination of dolutegravir (DTG) plus darunavir/ritonavir (DRV/r). A randomized clinical trial comparing the switch to this regimen towards the continuation of $\mathrm{DRV} / \mathrm{r}$ plus 2 nucleoside analogues is still recruiting patients [2]. When we started this strategy of dual rescue therapy or simplification of more complex rescue regimens, the OPTIONS study, the first randomized trial to utilize a web utility in combination with centralized expert opinion to guide the selection of salvage antiretroviral therapy was published [3]. The 413 participants had antiretroviral (ARV) experience or resistance to nucleoside reverse transcriptase inhibitors (NRTI) and non-NRTI (NNRTI), were taking a protease inhibitor (PI)containing regimen for at least 8 weeks prior to study entry, and had plasma HIV RNA $\geq 1000$ copies/mL. Enfuvirtide (ENF), etravirine (ETR), DRV, maraviroc (MVC), raltegravir (RAL), and tipranavir (TPV) plus ritonavir booster allowed 20 potential ARV regimens. Fiftythree subjects had obliged regimen choice due to poor phenotypic sensitivity score while 360 were randomized to add or not NRTIs. The resulting regimens were rather complex, with 3 to 6 drugs and twice-daily dosing, and generally contained DRV and RAL. The proportion of patients achieving viral suppression at 48 weeks $(<50$ HIV-1 RNA copies $/ \mathrm{mL}$ ) was $70.2 \%$ and $74.1 \%$ for the Omit NRTI and for the Add NRTI arms, respectively [4]. Prescribers were generally oriented to prefer simpler regimens.

The combination of elvitegravir/cobicistat/tenofovir alafenamide/emtricitabine plus $800 \mathrm{mg}$ DRV (two pills once daily) demonstrated superiority towards the continuation of baseline therapy in a randomized (2:1), open-label, switch trial in treatment-experienced virologically suppressed subjects [5]. The baseline regimens contained in median 5 pills per day and the primary endpoint was maintaining $<50 \mathrm{HIV}-1$ RNA copies/mL.

In this context some authors were looking forward for nucleoside-sparing regimens in the long-term care of $\mathrm{HIV}$ infection, welcoming the possible positive contribution of DTG [6].

The present study reports what the clinical practice had already realised, aiming for convenience, simplicity, potency and high genetic barrier, prescribing this combination to several patients and controlling them closely until viral suppression was achieved.

\section{Methods}

All subjects who had started DTG plus DRV/r between March 1, 2014 and September 30, 2015 were included in an Italian observational multicenter cohort named Tivista (Tivicay plus Prezista Observational cohort). After approval by Ethics Committees (EC) no further enrolment was allowed. Since the last publication two centres that were waiting for EC approval joined the group, increasing the population size. For homogeneity no data were collected after week 48 (plus 8 weeks' window as observational studies do not dictate strict timelines). The follow-up was censored at November, 30, 2016. Subjects were not required to be naive to DRV or DTG, but regimens containing both drugs were not included.

Participating subjects have signed an informed consent according to local procedures, the study has been approved by the coordinating centre and by all participating centres and conducted according to the Good Clinical Practice (GCP) Guidelines.

The aim of the study is to assess the efficacy of this approach. The primary end-point was the proportion of subjects achieving or maintaining virologic suppression $<50$ copies/mL at week 24. Secondary end points were maintaining virologic suppression in the follow-up (weeks 48 and 96) and safety, as proportion of drop-outs for any reason and grade 3-4 adverse events.

\section{Safety data and laboratory standards}

In this update it was decided to document the impact of the switch on some routine safety analyses. Investigators retrospectively reported to the coordinating center serum glucose, alanine aminotransferase (ALT), aspartate aminotransferase (AST), total cholesterol (TC), high density lipoprotein (HDL-C), low density lipoprotein (LDL-C) and triglycerides (TG). The estimated glomerular filtration rate (e-GFR) was calculated at baseline and at follow-up according to the Modification of Diet in Renal Disease (MDRD) equation [7]. Plasma viral suppression status was classified as harbouring $\geq 50 \mathrm{HIV}-1$ RNA copies/mL, or detectable below 50 copies $/ \mathrm{mL}$, or undetectable (no virus detected, NVD = 0 copies $/ \mathrm{mL}$ ).

Viremia was measured depending on centers with Abbot HIV-1 RT-PCR, threshold 37 copies, COBAS TaqMan HIV-1 test, threshold 20 copies, and Single Copy Assay. TruGene ${ }^{\mathrm{\tau m}}$ was used for resistance testing, validated on the Stanford Algorithm, and cumulative data was considered. Data were collected from electronic or hand-written patients' case record forms, according to each centre's organization and sent as pre-specified excel files. Available data from routine therapeutic monitoring of darunavir and dolutegravir trough plasma concentration were analysed.

For the toxicity analyses the Common Terminology Criteria for Adverse Events (CTCAE, Version 4.03, June 14, 2010) was considered [8]. Adverse clinical events and deaths were reported to the local Ethics Committees and authorities as required by the law. 


\section{Statistical analysis}

The statistical analysis was limited to routine parameters (median, mean and standard error) and to the paired $\mathrm{t}$ test for the comparison between the baseline and 48week metabolic and immunologic values. The virologic response is reported as the number of patients in each viral suppression category and as the median and interquartile range of $\log _{10}$ copies $/ \mathrm{mL}$ in those who do not reach suppression at each time point. The other analyses consider the on-treatment population. The sample size was obtained from the participants' adhesion.

\section{Results}

One hundred and thirty subjects were followed for a median of 56 months, mean 64 months. The median age was 52 years, females were $25.4 \%$ and non-Caucasians $8.9 \%$. The median baseline CD4+ T-cell count was 526/ mmc (IQR 329-718). Fifty-nine subjects had been exposed to INSTIs and all had been exposed to PIs. Eighty-one had darunavir in their pre-switch regimen and one had dolutegravir. The main risk factor for acquiring HIV was being male homosexual, 48.5\% $(n=63)$, followed by drug abuse, $26.9 \%(n=35)$ and heterosexual intercourse, $24.6 \%(n=32)$. Reasons that led to the switch were simplification, $44.6 \%(n=56)$, viral failure $30 \%(n=39)$ toxicity $16.9 \%(n=22$, of which 11 for osteopoenia/osteoporosis, 3 each for lipodystrophy and cardiovascular problems, 2 for renal toxicity and one each for gastrointestinal toxicity, diabetes and creatine kinase elevation), non-adherence, $4.6 \%(n=6)$, persistent low-level viremia, $3.1 \%(n=4)$, and drug-drug interaction, $0.8 \%(n=1)$. The algorithm of the study population is represented in Fig. 1.

At baseline 118 subjects had documented resistance to 1 to 5 ARV classes. For the remaining 12 patients, viral failure was not accompanied by a genotypic test. Twentythree subjects (17.7\%) had never experienced virologic failure but had transmitted drug resistance mutations.

One hundred and sixteen patients (89.2\%) had NRTI resistance-associated mutations (RAMs) at baseline, 98 (75.4\%) had NNRTI RAMs, 91 (70\%) had PI RAMs and 12 (10.6\%) had integrase strand transfer inhibitors (INSTI) RAMs (See Table 1).

Twenty subjects had reduced baseline sensitivity to darunavir (Stanford median score 15, range 15-40), and 12 had reduced sensitivity to INSTIs (Stanford median score 10, range 10-25) but none rebounded. None developed new drug resistance mutations during the study.

Of those subjects who had resistance to 3-4 drug classes $(n=70), 35$ were not failing at baseline, but rather simplified from raltegravir and boosted darunavir-based three- $(n=31)$ or four-drugs regimens $(n=9)$.

One hundred and thirteen have started the combination of DRV $800 \mathrm{mg}$ plus RTV $100 \mathrm{mg}$ plus DTG $50 \mathrm{mg}$ every $24 \mathrm{~h}, 3$ DRV $600 \mathrm{mg}$ plus RTV $100 \mathrm{mg}$ every $12 \mathrm{~h}$ and 14 DRV 600 mg plus RTV 100 mg plus DTG 50 mg every $12 \mathrm{~h}$. All of those who were on BID therapy had active HIV-1 replication at baseline (range 5.46-6.16 $\log _{10}$ HIV1 RNA copies $/ \mathrm{mL}$ ). All of them suppressed viremia to $<50$ copies/mL during the follow-up.

Between week 24 and 48 one subject was lost to follow-up, one interrupted the regimen for liver

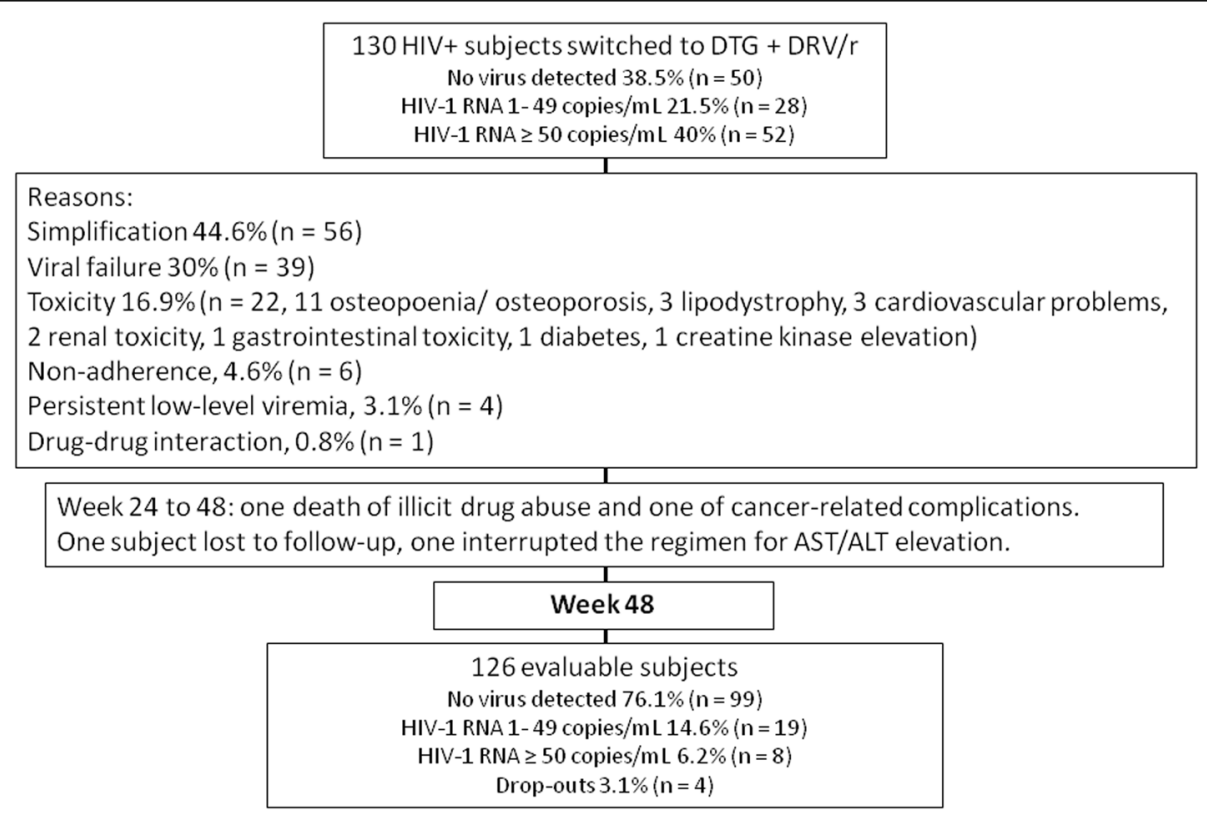

Fig. 1 Algorithm of the study population. DTG = dolutegravir; DRV/r = darunavir boosted with ritonavir; AST = aspartate aminotransferase; $\mathrm{ALT}=$ alanine aminotransferase 
Table 1 Baseline HIV-1 drug resistance mutations in the study population

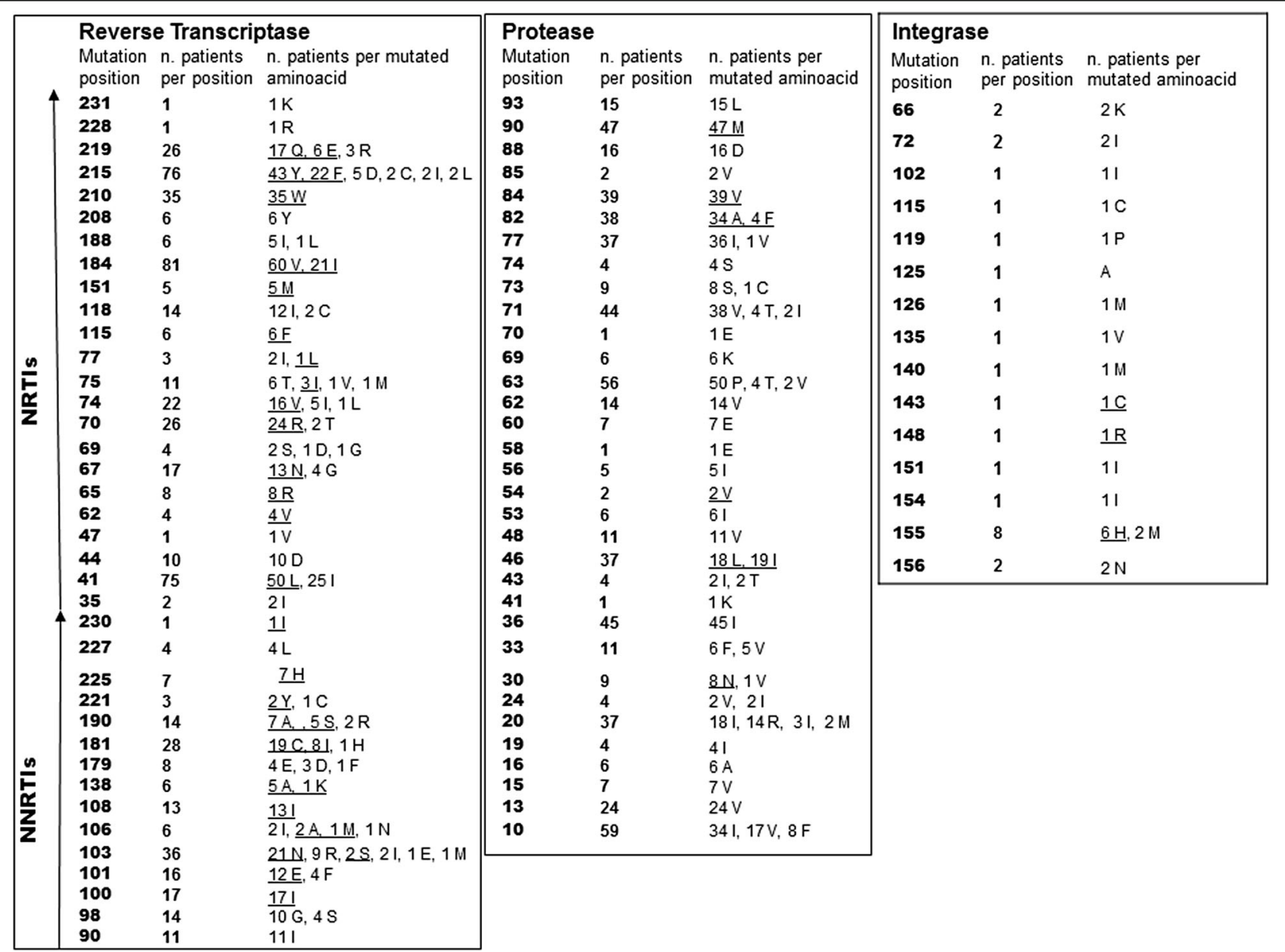

Primary resistance mutations (Wensing, 2017) are underlined

enzymes' elevation, one died of illicit drug abuse and one of cancer-related complications.

The proportion of subjects harboring active HIV replication dropped from $40 \%$ at baseline to $6.1 \%$ by week 48 , while those who had NVD at real-time polymerase chain reaction (RT-PCR) increased from $38.5 \%$ to $76.2 \%$. Overall, at baseline 78 subjects had $<50$ HIV-1 RNA copies/mL $(60 \%)$ and this proportion increased at week 48 to $90,8 \%(n=118)$ At week 48,19 subjects $(14,6 \%)$ had HIV RNA between 1 and 49 copies $/ \mathrm{mL}$ and eight subjects $(6,1 \%)$ had $\geq 50$ HIV- 1 RNA copies $/ \mathrm{mL}$. Of the latter, 3 had a slow but constant decay from median baseline 5.12 to $2.30 \log _{10}$ HIV-1 RNA copies/mL, 3 had at least three-class drug resistance with a Stanford DRV score > 60 and median viral decay from 4.39 to 1.75 $\log _{10}$ HIV-1 RNA copies/mL, and 2 had dropped out of therapy 4 and 7 days before blood sampling. Considering separately two populations, those who started with active HIV replication $(\geq 50$ copies $/ \mathrm{mL}$ ) and those who started with $0-49$ copies $/ \mathrm{mL}$, at week 48 the success rate was $88.5 \%$ in those who had started with active replication, as 3 with high baseline viral load and 3 with extensive baseline resistance, had still very low but detectable viremia, while in those who started with 0-49 copies/ $\mathrm{mL}$ the success rate was $97.4 \%$ as in this group two subjects were not on therapy on the sampling day, as described above. Of interest, both were in the subgroup starting with 1-49 copies/mL, as some authors suggest that this low but persistent viremia may sometime hide adherence problems. The mean viral load in this population at week 48 was $1.89 \log _{10}$ copies/mL (range 1.70 2.57, Fig. 2). The CD4+ T-cell absolute count and proportion increased by 27 cells $/ \mathrm{mm}^{3},+5 \%$, not reaching statistical significance.

A single-point pharmacokinetic analysis in a subgroup of 32 subjects confirmed the safety of the association: DTG median $C_{\text {trough }}$ was $579 \mathrm{ng} / \mathrm{mL}$ (range 275-5036), while DRV median $\mathrm{C}_{\text {trough }}$ was $3007 \mathrm{ng} / \mathrm{mL}$ (range 6788053) and no values were found to be below the safety threshold. Five subjects were taking DRV $600 \mathrm{mg}$ twice daily and had $\mathrm{C}_{\min }$ above the threshold for resistant strains, and three were taking DTG twice daily. 


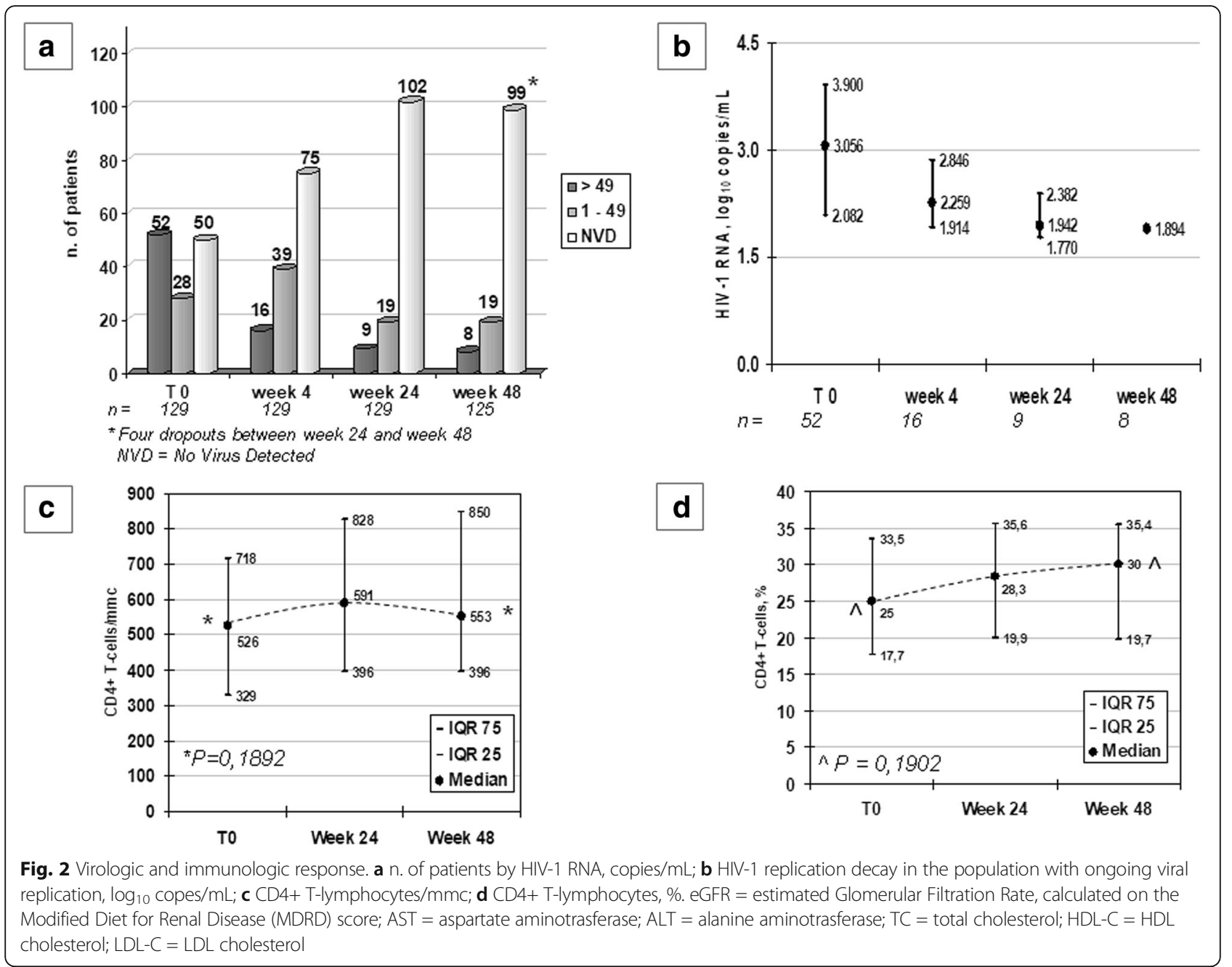

The proportion of subjects having any metabolic alterations at baseline decreased for serum glucose, creatinine, MDRD $<90 \mathrm{~mL} / \mathrm{min}$, ALT, AST, total-, HDL- and LDL-cholesterol and triglycerides. The proportion of subjects having MDRD $<60$ remained stable at $4.6 \%$. Overall 84/175 (48\%) baseline laboratory alterations returned to normality. Considering each parameter in the whole population, none varied significantly (Fig. 3).

In particular, the median variation in serum creatinine was $-0,03$ and the eGFR decrease on average by $2.66 \mathrm{~mL} / \mathrm{min}$. None of the subjects who had altered serum creatinine at baseline showed progression and 6 out of 8 improved, having discontinued tenofovir.

Overall this strategy yielded mean savings for $€$ $169,428.00$ considering the whole population, the observation period and the dropouts. The figure was generated only from the cost of the previous and current antiretroviral regimen.

\section{Discussion}

While awaiting the results of the randomized DUALIS study [2], our data suggest that the combination of DTG plus DRV/r may be considered reliable in salvage or simplification of salvage regimens. The pharmacokinetic interaction between the two drugs [9] did not seem to impact neither causing subtherapeutic $\mathrm{C}_{\min }$ in the subjects tested nor leaving space for plasma HIV-1 RNA rebound in any subject. In particular, $70 \%$ of our patients would not have been suitable for the combination of DTG plus atazanavir, which has a favourable interaction profile [10] due to baseline resistance to the latter and it should be said that clinical experience with this combination is still limited though promising. Moreover, we have not reported significant neurological side effects, as suggested by Hoffman et al. [11] Possible reasons may be the combination, which does not include abacavir, the fact that many of our patients had received the indication to take the drug in the morning and the relatively small number of other at risk subjects (15 elderly and 33 women). 


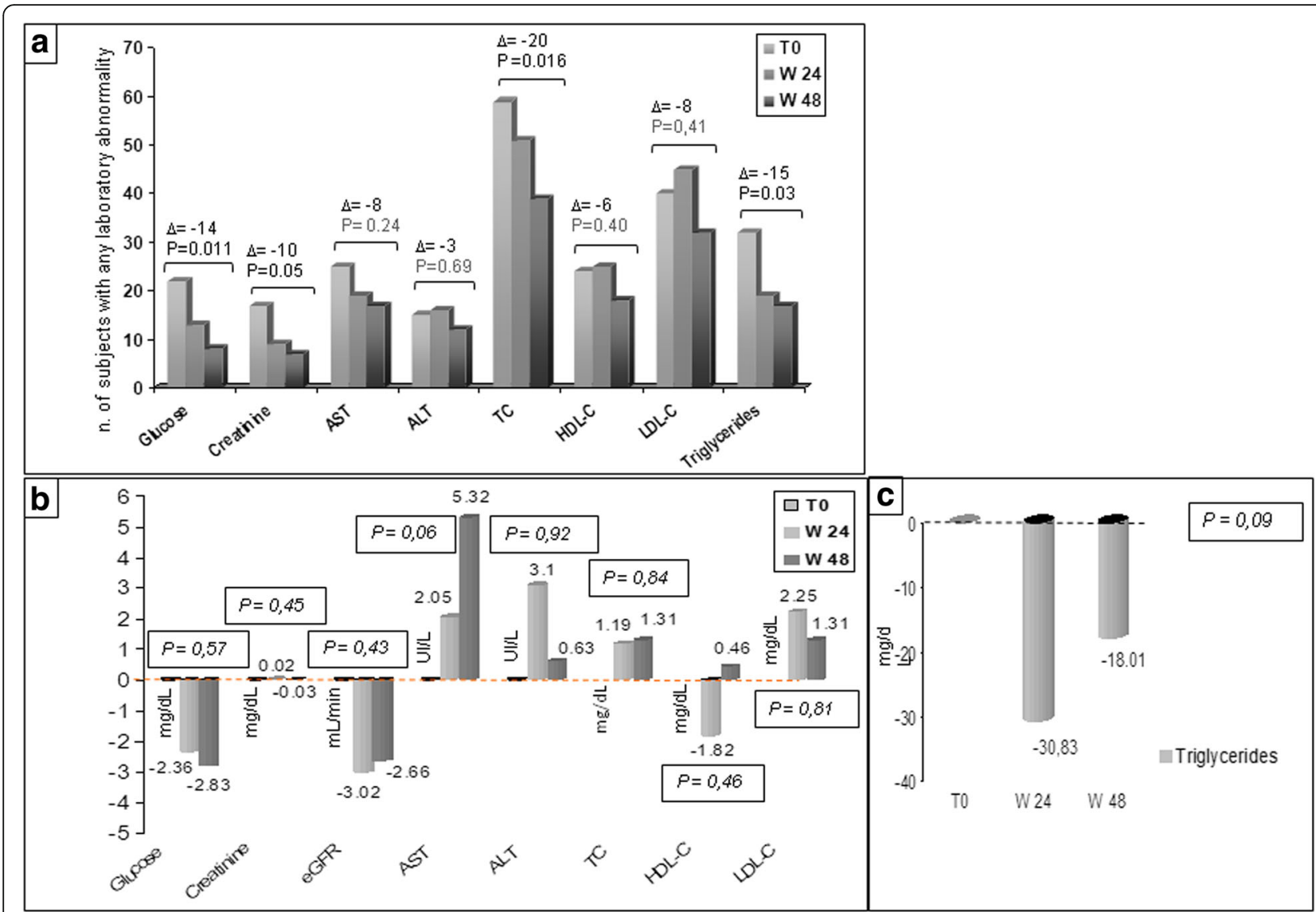

Fig. 3 Metabolic profile during the follow-up. a Number of subjects with any laboratory abnormality at baseline, week 24 and week 48 . b Variation of metabolic indicators from baseline, mean values; $\mathbf{c}$ Variation in triglycerides (different scale). AST = Aspartate Aminotransferase; ALT = Alanine Aminotransferase; HDL-C = High-Density Lipoprotein - Cholesterol; LDL-C = Low-Density Lipoprotein - Cholesterol; MDRD = Modified Diet for Renal Disease; $\mathrm{TC}=$ Total Cholesterol

The study limitations include poor patient selection (the only two inclusion criteria were being HIV-1 antibody positive and having started DTG plus DRV/r by the end of September 2015), leading to the inclusion of a heterogeneous population, made out of failures and simplification, once- and twice-daily regimens, adherent and poorly adherent subjects and subjects harbouring treatmentresistant and treatment-sensitive strains. The set of metabolic parameters furthermore reflects standard follow-up data shared by centres and does not include bone density measures nor inflammatory markers.

\section{Conclusions}

Switching to DTG plus DRV/r provided a simple and safe rescue regimen to all subjects, controlling viral replication in a high proportion of patients. The metabolic impact was favourable in a proportion of subjects who had baseline alterations, while no significant modifications were observed in mean and median values when also normal baseline values were included.

\section{Additional file}

Additional file 1: Tivista Study Week 48 database. (TIFF 31 kb)

\section{Abbreviations}

ALT: Alanine aminotransferase; ARV: Antiretroviral; AST: Aspartate aminotransferase; CD4: Cluster difference 4; CTCAE: Common Terminology Criteria for Adverse Events; $C_{\text {trough: }}$ Trough concentration; DRV/r: Darunavir boosted with ritonavir; DTG: Dolutegravir; EC: Ethics Committee; eGFR: Estimated glomerular filtration rate; ENF: Enfivirtide; ETV: Etravirine; GCP: Good clinical practice; HDL: High-density lipoprotein; HIV-1: Human immunodeficiency virus type 1; LDL: Low density lipoprotein; MDRD: Modified diet for renal disease; MVC: Maraviroc; NNRTI: Nonnucleoside reverse transcriptase inhibitor; NRTI: Nucleoside reverse transcriptase inhibitor; NVD: No virus detected; PI: Protease inhibitor; RAL: Raltegravir; RNA: Ribonucleic acid; RT-PCR: Real-time Polymerase Chain Reaction; TPV: Tipranavir

\section{Acknowledgements}

We acknowledge Mrs. Maureen Naomi Quinn for reviewing this text as native american health worker.

\section{Funding}

This research did not receive any specific grants from funding agencies from funding agencies in the public, commercial or not-for-profit sectors. 


\section{Availability of data and materials}

The study database is attached as Additional file 1.

\section{Authors' contributions}

AFC planned and proposed the study, contributed participants' data, analyzed them and drafted the paper. MVC clinically followed, enrolled and contributed patients' data, coordinated the team and in particular dealt with Ethics' Committees and revised the paper with minor comments. GCO, GC, AMC, NR, GMB, FPPN and GB clinically followed, enrolled and contributed patients' data and revised the paper with minor comments. GS, GVDS, AS, SR contributed data and revised the draft, providing substantial improvement. GR supervised the team's work and contributed clinical advice during the follow-up. All authors read and approved the final manuscript.

\section{Ethics approval and consent to participate}

The study was approved by the coordinating centre's Ethics Committee and subsequently by all the ECs of the participating centres.

List of the Ethics' Committees:

Comitato Etico Interaziendale Milano Area A (Protocol n. 6926/2016).

Comitato Etico delle Aziende Sanitarie dell'Umbria, Perugia.

Comitato Etico della ASL To/2 di Torino.

Comitato Etico per la Sperimentazione dell'Azienda Ospedaliera di Padova. Comitato Etico Regionale Regione Liguria.

Comitato Etico Sperimentazione Clinica Azienda Ospedaliera-Universitaria Careggi, Firenze

Comitato Etico dell'Università Cattolica del Sacro Cuore - Policlinico

Universitario Agostino Gemelli, Roma.

Comitato Etico IRCCS Policlinico San Matteo, Pavia.

Comitato Etico Brianza.

The patients signed a general privacy agreement for data management and an informed consent for accepting a non-conventional regimen in accordance with local procedures. Since the data collection was retrospective-prospective, no study-specific informed consent exist since at the time of the switch no study was planned.

\section{Consent for publication}

Not applicable

\section{Competing interests}

The authors declare that they have no competing interests.

\section{Publisher's Note}

Springer Nature remains neutral with regard to jurisdictional claims in published maps and institutional affiliations.

\begin{abstract}
Author details
${ }^{1}$ 1st Division of Infectious Diseases, ASST Fatebenefratelli-Sacco, Via Giovanni Battista Grassi, 74, pavillion 56, Malattie Infettive, 2nd floor, 20157 Milan, Italy. ${ }^{2} 1$ st Division of Infectious Diseases Amedeo di Savoia Hospital, Torino, Italy. ${ }^{3}$ Division of Infectious Diseases, "Careggi" Hospital, Firenze, Italy. ${ }^{4}$ Division of Infectious Diseases, Ospedali Galliera, Genova, Italy. ${ }^{5}$ Infectious Diseases Clinic, Azienda Ospedaliero-Universitaria di Perugia, Perugia, Italy. ${ }^{6}$ Infectious and Tropical Diseases, Azienda Ospedaliera-Universitaria di Padova, Padova, Italy. ${ }^{7}$ Clinic of Infectious Diseases, San Gerardo Hospital, ASST Monza, University of Milano-Bicocca, Monza, Italy. ${ }^{8}$ Infectious Diseases Clinic, DIBIC Luigi Sacco, University of Milano, Milano, Italy. ${ }^{9}$ Infectious Diseases Clinic, "San Martino" Hospital, Genova, Italy. ${ }^{10} 2$ nd Division of Infectious Diseases, Università Cattolica del Sacro Cuore, Rome, Italy. ${ }^{11}$ 2nd Division of Infectious Diseases, "Policlinico San Matteo" Hospital, Pavia, Italy. ${ }^{12}$ Whitwaterstrand University, Johannesburg, South Africa.
\end{abstract}

Received: 21 June 2017 Accepted: 21 September 2017

Published online: 30 September 2017

\section{References}

1. Capetti AF, Sterrantino G, Cossu MV, Cenderello G, Cattelan AM, De Socio GV, et al. Correction: Correction: Salvage therapy or simplification of salvage regimens with dolutegravir plus ritonavir-boosted darunavir dual therapy in highly cART-experienced subjects: an Italian cohort. Antivir Ther. 2017;22(3): 273-5. doi: 10.3851/IMP3109. Epub 2016 Nov 28
2. Dual Therapy With Boosted Darunavir + Dolutegravir (Dualis) https://clinicaltrials. gov/ct2/show/NCT02486133 Accessed 4 April, 2017.

3. Tashima KT, Mollan KR, Na L, Gandhi RT, Klingman KL, Fichtenbaum CJ, et al. Regimen selection in the OPTIONS trial of HIV salvage therapy: drug resistance, prior therapy, and race-ethnicity determine the degree of regimen complexity. HIV Clin Trials. 2015;16(4):147-56.

4. Tashima KT, Smeaton LM, Fichtenbaum CJ, Andrade A, Eron JJ, Gandhi RT, et al. HIV salvage therapy does not require nucleoside reverse transcriptase inhibitors: a randomized trial. Ann Intern Med. 2015;163(12):908-17.

5. Huhn GD, Tebas P, Gallant J, Wilkin T, Cheng A, Yan M, et al. A randomized, open-label trial to evaluate switching to elvitegravir/cobicistat/emtricitabine/ tenofovir alafenamide plus darunavir in treatment-experienced HIV-1 infected adults. J Acquir Immune Defic Syndr. 2017;74(2):193-200.

6. Pasquau J, Hidalgo-Tenorio C. Nuke-Sparing Regimens for the Long-Term Care of HIV Infection. AIDS Rev. 2015;17:220-30.

7. Levey AS, Coresh J, Greene T, Marsh J, Stevens LA, Kusek JW, et al. Chronic Kidney Disease Epidemiology Collaboration. Expressing the Modification of Diet in Renal Disease Study Equation for Estimating Glomerular Filtration Rate with Standardized Serum Creatinine Values. Clin Chem. 2007;53(4):766-72.

8. CTCAE website. Available: http://evs.nci.nih.gov/ftp1/CTCAE/About.html. Accessed 21 February 2017.

9. Cottrell ML, Hadzic T, Kashuba ADM. Clinical Pharmacokinetic Pharmacodynamic and Drug-Interaction Profile of the Integrase Inhibitor Dolutegravir. Clin Pharmacokinet. 2013;52(11):981-94.

10. Cattaneo D, Minisci D, Cozzi V, Riva A, Meraviglia P, Clementi E, et al. Dolutegravir plasma concentrations according to companion antiretroviral drug: unwanted drug interaction or desirable boosting effect? Antivir Ther. 2016; doi: 10.3851/IMP3119. [Epub ahead of print]

11. Hoffmann C, Welz T, Sabranski M, et al. Higher rates of neuropsychiatric adverse events leading to dolutegravir discontinuation in women and older patients. HIV Med. 2017:18(1):56-63.

\section{Submit your next manuscript to BioMed Central and we will help you at every step:}

- We accept pre-submission inquiries

- Our selector tool helps you to find the most relevant journal

- We provide round the clock customer support

- Convenient online submission

- Thorough peer review

- Inclusion in PubMed and all major indexing services

- Maximum visibility for your research

Submit your manuscript at www.biomedcentral.com/submit
Biomed Central 